\title{
Direct endoscopy and diagnosis of adenocarcinoma following metal stent-based drainage of a pancreatic cyst
}
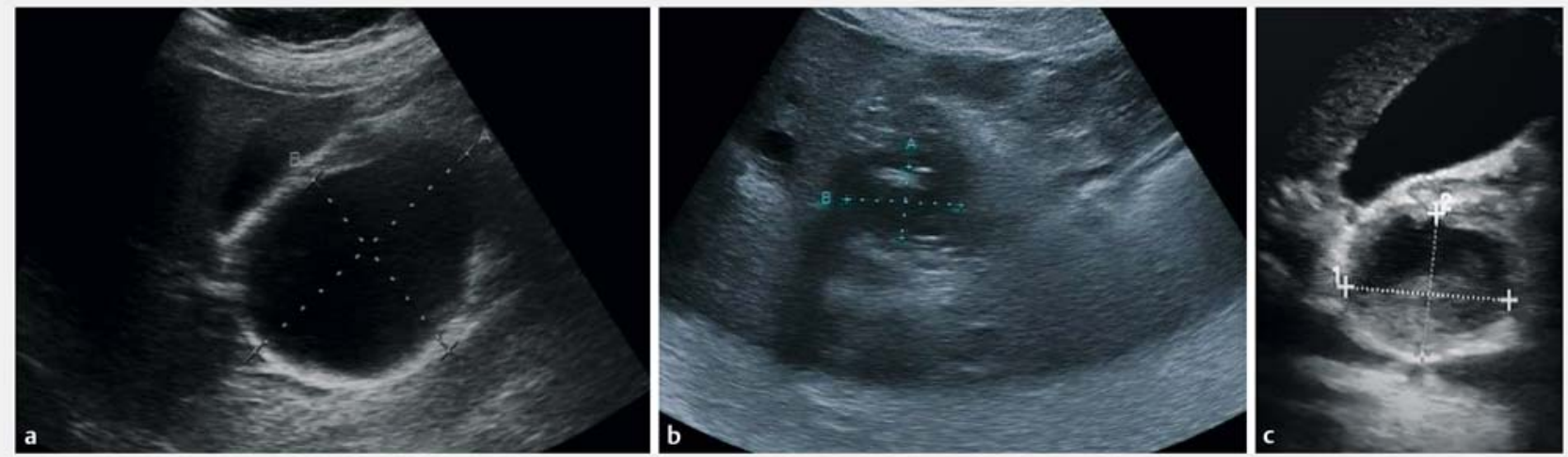

- Fig. 1 Abdominal ultrasound. a Large cystic structure in the pancreatic head. b At 4 weeks after drainage, only a small cyst residue was detected. c Signs of cyst infection 5 months later.

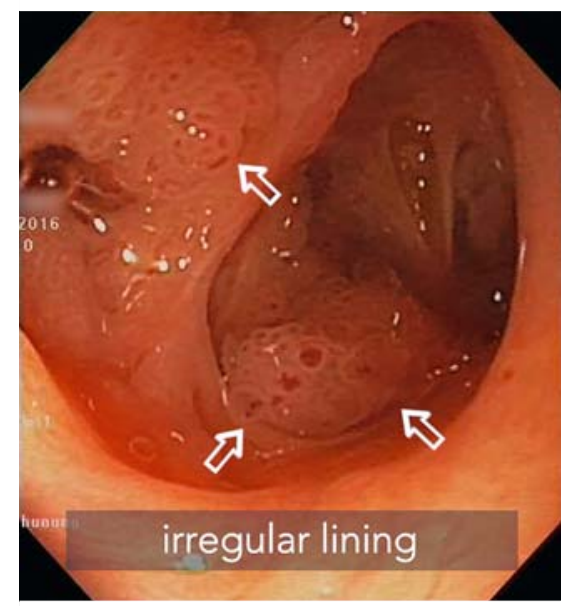

Fig. 2 Irregular internal surface of the cystic structure.

Abdominal ultrasound in a 71-year-old man, 6 months after acute alcoholic pancreatitis, showed a pancreatic cystic structure $(4 \mathrm{~cm})$, which was diagnosed as a pancreatic pseudocyst (PPC). Drainage was not performed as the patient was asymptomatic. The PPC showed no size progression at 6-monthly follow-up abdominal ultrasound scans. CA19-9 was normal.

At 10 years after initial PPC detection, the patient, who had not consumed alcohol for years, presented with gastric

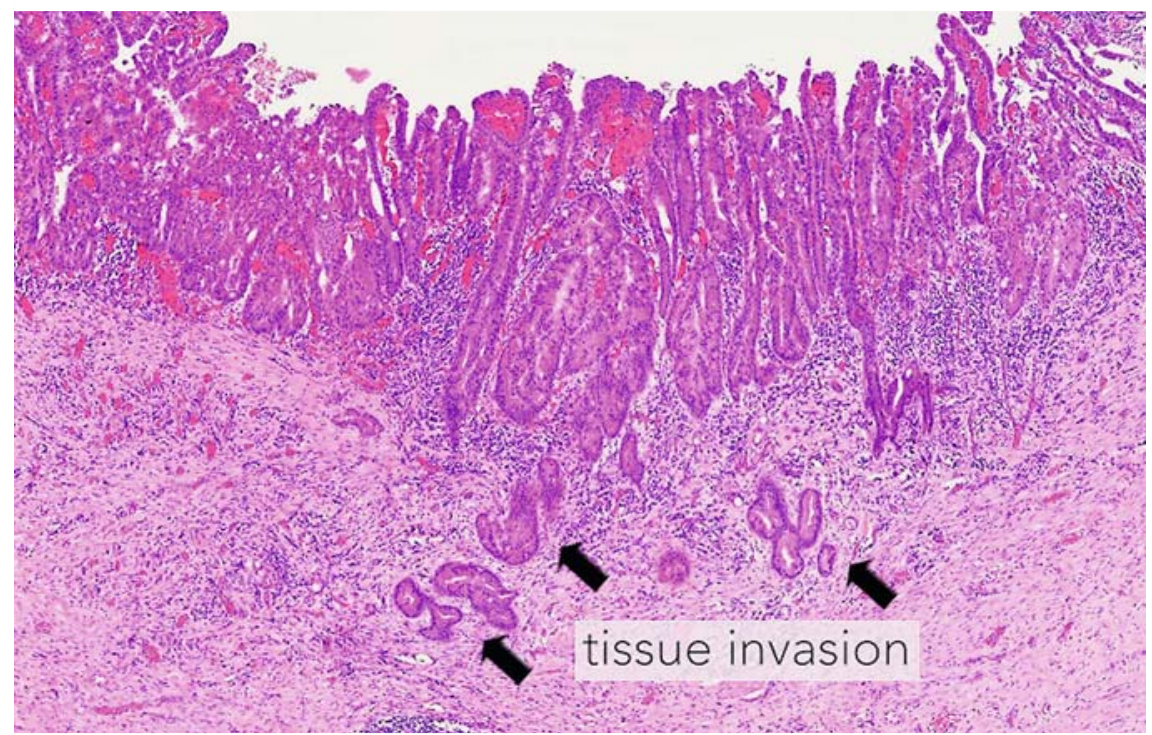

- Fig. 3 Hematoxylin and eosin staining ( $\times 50)$, demonstrating intraductal papillary mucinous neoplasm with focal progression to invasive carcinoma.

outlet obstruction and cyst size progression ( Fig.1a). Cystogastrostomy was performed using a lumen-apposing selfexpandable metal stent (LASEMS), which led to release of clear fluid. Abdominal ultrasound and endoscopy 4 weeks later showed cyst regression ( Fig. $\mathbf{1}$ b), and the LASEMS was therefore removed. However, 5 months later, the patient be- came symptomatic with abdominal pain, fever, and a rise in $\mathrm{C}$-reactive protein and CA19-9 (85 U/mL). Ultrasound indicated cyst infection ( $\mathbf{F i g} \cdot \mathbf{1}$ c). Cystoduodenostomy using an LASEMS revealed a putrid cyst content. Subsequently, the cyst lumen was inspected through the LASEMS ( $>$ Video 1 ), revealing an irregular cyst surface and branch duct involve- 


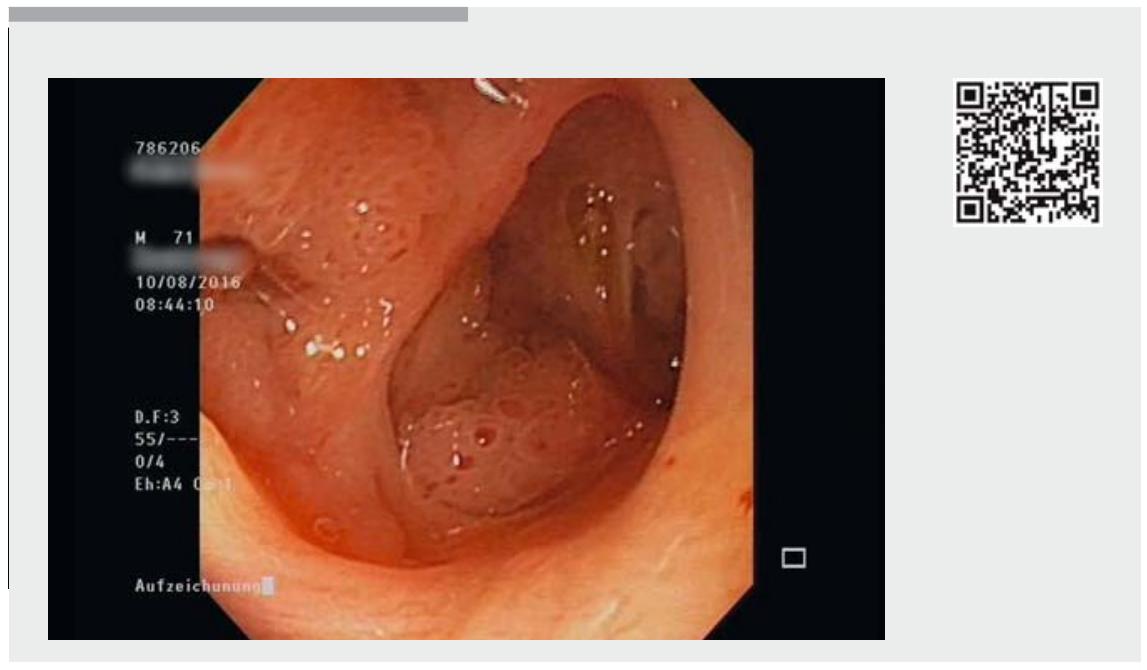

Video 1 Diagnosis of branch duct intraductal papillary mucinous neoplasm, with focal progression to invasive carcinoma, by lumen-apposing self-expandable metal stent-based drainage of a cystic structure that was previously diagnosed as a pancreatic pseudocyst.

ment ( $\triangleright$ Fig. 2). Histopathological analysis of biopsies from the cyst wall showed an intraductal papillary mucinous neoplasm (IPMN), with focal progression to invasive carcinoma, which was confirmed after Whipple resection (pT1pN0M0; - Fig. 3). Postoperative surveillance for 1 year showed no signs of disease recurrence.

PPCs can be difficult to distinguish from IPMN, particularly in cases of unilocular cysts and history of pancreatitis [1-3]. Here, we report a case of branch duct IPMN mimicking a PPC, in which drainage by an LASEMS and endoscopic inspection revealed pancreatic adenocarcinoma. This case highlights potential benefits of drainage of pancreatic fluid collections by LASEMS, which facilitates direct cyst endoscopy and can assist in the detection of malignancy. Moreover, these data lend support to a low threshold of endoscopic cyst inspection after LASEMS-based drainage of pancreatic fluid collections.

Endoscopy_UCTN_Code_CCL_1AF_2AZ_3AB
[1] Kang SH, Kim KH, Kim TN. A case of branch duct intraductal papillary mucinous neoplasm mimicking pseudocysts complicated by recurrent pancreatitis. Korean J Pancreas Biliary Tract 2015; 20: $94-98$

[2] Barresi L, Tarantino I, Granata A et al. Pancreatic cystic lesions: how endoscopic ultrasound morphology and endoscopic ultrasound fine needle aspiration help unlock the diagnostic puzzle. World J Gastrointest Endosc 2012; 4: 247-259

[3] Sahani DV, Kadavigere R, Saokar A et al. Cystic pancreatic lesions: a simple imagingbased classification system for guiding management. RadioGraphics 2005; 25: $1471-1484$

\section{Bibliography}

DOI https://doi.org/10.1055/s-0043-124182

Published online: 12.1.2018

Endoscopy 2018; 50: E72-E73

(c) Georg Thieme Verlag KG

Stuttgart · New York

ISSN 0013-726X

None

The authors

Sebastian Zeissig ${ }^{1}$, Renate Schmelz ${ }^{1}$, Stefan Brückner ${ }^{1}$, Daniela E. Aust ${ }^{2}$, Gustavo B.

Baretton $^{2}$, Jochen Hampe ${ }^{1}$

1 Medizinische Klinik I, Universitätsklinikum Carl Gustav Carus, Technische Universität Dresden, Dresden, Germany

2 Institut für Pathologie, Universitätsklinikum Carl Gustav Carus, Technische Universität Dresden, Dresden, Germany

\section{Corresponding author}

\section{Sebastian Zeissig, MD}

Medizinische Klinik I, Universitätsklinikum Carl Gustav Carus, Technische Universität Dresden, Fetscherstr. 74, 01309 Dresden, Germany Fax: +49-351-45882309 sebastian.zeissig@tu-dresden.de

\section{ENDOSCOPY E-VIDEOS}

https://eref.thieme.de/e-videos

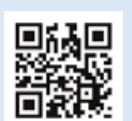

Endoscopy E-Videos is a free access online section, reporting on interesting cases and new techniques in gastroenterological endoscopy. All papers include a high quality video and all contributions are freely accessible online.

This section has its own submission website at https://mc.manuscriptcentral.com/e-videos 